
\title{
25 Research Sourere \\ Prevalence and Risk Factors for Gastroesophageal Reflux in Symptomatic Very Preterm Neonates
}

\author{
Yu Hu \\ Shengjing Hospital of China Medical University \\ Hua Fu \\ Shengjing Hospital of China Medical University \\ Cheng Zhang ( $\nabla$ zhangshucheng76@126.com ) \\ Shengjing Hospital of China Medical University
}

\section{Research Article}

Keywords: multichannel intraluminal impedance, gastroesophageal reflux disease, infant, bronchopulmonary dysplasia, apnea

Posted Date: November 20th, 2020

DOI: https://doi.org/10.21203/rs.3.rs-111467/v1

License: (c) (i) This work is licensed under a Creative Commons Attribution 4.0 International License. Read Full License 


\section{Abstract}

Objectives Because of immature development, preterm infants are prone to respiratory and digestive symptoms, such as dyspnea, vomiting, and aspiration pneumonia. These symptoms are often observed in infants with gastroesophageal reflux (GER). However, the relationship between GER and these symptoms remains unclear.

Methods A cohort of 61 preterm infants born at 32 weeks of gestation or earlier with respiratory or digestive symptoms were retrospectively reviewed. All the preterm infants underwent 24-hour pHmultichannel intraluminal impedance monitoring. Clinical data were analyzed using logistic regression analyses.

Results Among the 61 symptomatic preterm infants, 34 were positive for pathologic GER with a prevalence of $55.7 \%$. The GER-associated symptoms included apnea (42.6\%), cyanosis (19.7\%), vomiting (14.8\%), pneumonia (9.8\%), and poor weight gain (13.1\%). These respiratory and digestive symptoms were more frequent in infants with pathologic GER than in those without $(p<0.05)$. The risk factors for GER included severe bronchopulmonary dysplasia (odds ratio [OR], 6.890; 95\% confidence interval [CI], 1.125-42.209). Probiotics and delayed full oral feeds reduced the risk of GER (OR, $0.642 ; 95 \% \mathrm{Cl}, 0.457-$ $0.901, p<0.05 ;$ OR, $0.234,95 \% \mathrm{Cl}, 0.069-0.794, \mathrm{p}<0.05)$.

Conclusions The prevalence of GER is high in symptomatic preterm infants. GER aggravates the respiratory and digestive symptoms; however, such symptoms are not always GER-associated. Probiotics and delayed full oral feeds can decrease the prevalence of GER.

\section{Introduction}

Gastroesophageal reflux (GER) is common in infants because of physiological factors, such as immaturity of the lower esophageal sphincter (LES), supine positioning, and frequent feeding. It was initially thought that approximately $70 \%-85 \%$ of infants with regurgitation required no intervention within the first 2 months of life (1). However, infants with GER may have symptoms, such as vomiting, poor weight gain, dyspnea, decreased oxygen saturation, and aspiration pneumonia. The presence of these conditions has been defined as gastroesophageal reflux disease (GERD) (2). Improvements in medical technology used for infant care have allowed for the survival of an increasing number of preterm infants and infants with very low birth weight (BW). More than $80 \%$ preterm infants may experience the clinical symptoms of GERD. However, diagnosing GER is difficult; therefore, the incidence of GER and its effects on infants are controversial.

Traditionally, 24-hour pH monitoring has been the gold standard technique for diagnosing GER. However, it cannot diagnose nonacidic episodes with $\mathrm{pH} \otimes 4$, which occur in infants because continuous feedings dilute the gastric acid. Mitchell et al. (3) reported that the stomach has a $\mathrm{pH} \otimes 4$ during only approximately $24.5 \%$ of the total time, with that rate ranging from $0.6 \%$ to $69.1 \%$. Because nonacid reflux is the most common type of reflux for infants, the multichannel intraluminal impedance (MII) technique, which 
overcomes the disadvantages of traditional 24-hour $\mathrm{pH}$ monitoring, has become a valid method used to diagnose GERD in preterm infants (4). With this method, the diagnostic accuracy has been significantly improved. This study aimed to investigate symptomatic preterm infants using gastric and esophageal 24hour pH-MII monitoring to determine the prevalence, clinical features, and risk factors of GER in very preterm infants.

\section{Results}

\section{Demographics}

Sixty-one preterm infants (33 male and 28 female infants) with a median GA of $30 \pm 1.28$ weeks (range, $26^{+5}$ weeks to 32 weeks) and a mean BW of $1409 \pm 362 \mathrm{~g}$ were reviewed. They received complete oral feeds at a median postmenstrual age (PMA) of $35 \pm 2.1$ weeks. All patients remained clinically stable and accepted the monitoring well. No one received medication for GER during hospitalization.

\section{GER prevalence in symptomatic preterm infants}

Among the 61 infants with symptoms, 34(16 female and 18 male infants, 56\%) were GER-positive and 27 (44\%) were GER-negative. Eighteen (53\%) cases of acid reflux and $16(47 \%)$ cases of other reflux (including weakly acidic and nonacidic reflux) occurred in GER-positive infants. Table 1 presents the GA and BW. There was no difference between the GER-positive and GER-negative groups classified by GA and $B W$ ( $p=0.886$ and 0.538 , respectively). A similar result was found between the acid reflux group and other reflux group classified by GA and BW ( $p=0.797$ and 0.052 , respectively).

Table 1. Cohort characteristics of patients $(n=61)$

\begin{tabular}{|c|c|c|c|c|c|}
\hline \multirow[t]{2}{*}{ Factors } & \multirow[t]{2}{*}{ Grade } & \multicolumn{3}{|c|}{ GER positive, n (\%) } & \multirow[t]{2}{*}{ GER negative } \\
\hline & & acid reflux & other reflux & total & \\
\hline \multirow[t]{3}{*}{ GA } & $<28 w$ & $1(5.6 \%)$ & $2(12.5 \%)$ & $3(8.8 \%)$ & $2(7.4 \%)$ \\
\hline & $28-30 w$ & $7(38.9 \%)$ & $6(37.5 \%)$ & $13(38.2 \%)$ & $13(48.2 \%)$ \\
\hline & $30-32 w$ & $10(55.6 \%)$ & $8(50 \%)$ & $18(53 \%)$ & $12(44.4 \%)$ \\
\hline \multirow[t]{3}{*}{ BW } & $<1000 \mathrm{~g}$ & $1(5.6 \%)$ & $5(31.3 \%)$ & $6(17.6 \%)$ & $3(11.1 \%)$ \\
\hline & $1000-1500 \mathrm{~g}$ & $6(33.3 \%)$ & $7(43.7 \%)$ & $13(38.2 \%)$ & $14(51.9 \%)$ \\
\hline & $>1500 \mathrm{~g}$ & $11(61.1 \%)$ & $4(25 \%)$ & $15(44.2 \%)$ & $10(37 \%)$ \\
\hline
\end{tabular}

GA: gestational age, BW: birth weight

Among the 34 infants GER-positive for GER, 53\% (18/34) had acid reflux, 26.5\% (9/34) had weakly acidic reflux, and $20.6 \%(7 / 34)$ had nonacidic reflux. Acid reflux-positive preterm infants (10 male and 8 female infants) born at $30.12 \pm 0.97$ weeks of gestation (range, $28-31^{+6}$ weeks of gestation) had an average 
weight of $1530.83 \pm 266.35 \mathrm{~g}$ (range, 973-1900 g). Eight male and eight female GER-positive infants with weak acidic or nonacidic reflux were born at $29.78 \pm 1.53$ weeks of gestation (range, $26^{+5}-32$ weeks of gestation) and had an average weight of $1336.63 \pm 482.16 \mathrm{~g}$ (range, 695-2355 g).

\section{Relationship between GER and the symptoms}

Among the 61 infants, $26(42.6 \%)$ had apnea, 12 (19.7\%) had cyanosis, 9 (14.8\%) had vomiting, 6 (9.8\%) had pneumonia, and $8(13.1 \%)$ had poor weight gain. By SSI and SAP analysis, the GER-associated symptoms included apnea (52.9\%), cyanosis (17.6\%), vomiting (8.8\%), pneumonia (5.9\%), and poor weight gain (14.7\%). There was no difference in the aforementioned reflux-related symptoms for those in the GER-negative and GER-positive groups according to the chi-square test (Table 2). However, these symptoms were more frequent in the GER-positive group than those in the GER-negative group, indicating that GER might aggravate these symptoms $(p<0.05)$.

Table 2. Relationship between GER and the symptoms

\begin{tabular}{lllll}
\hline Symptoms & GER negative, n (\%) & GER positive, n (\%) & $\mathrm{t} / \mathbf{\chi}^{2}$ & $P$ \\
\hline apnea & $8(29.6)$ & $18(52.9)$ & 3.344 & 0.067 \\
cyanosis & $6(22.2)$ & $6(17.6)$ & 0.199 & 0.655 \\
vomiting & $6(22.2)$ & $3(8.8)$ & 2.148 & 0.143 \\
pneumonia & $4(14.8)$ & $2(5.9)$ & 1.354 & 0.245 \\
poor weight gain & $3(11.1)$ & $5(14.7)$ & 0.171 & 0.680 \\
\hline
\end{tabular}

\section{Risk factors for GER in the symptomatic preterm infants}

As shown in Table 3, there were no significant differences in the general conditions and clinical characteristics, including sex, GA, BW, Apgar score at 1 minute, PMA at the time of the study, duration of partial parenteral nutrition, caffeine administration, duration of mechanical ventilation, length of hospital stay, bronchopulmonary dysplasia (BPD), necrotizing enterocolitis (NEC), and nosocomial infection, for the GER-negative and GER-positive groups according to the independent samples t test (all $p>0.05)$. The durations of noninvasive auxiliary ventilation were statistically significant for the GER-negative and GERpositive groups $(14.67 \pm 9.1$ vs. $8.71 \pm 7.9, p<0.01)$. Infants in the GER-positive group had earlier full oral feeds than those of the GER-negative group $(35.18 \pm 2.16$ vs. $36.26 \pm 1.45, p<0.01)$. More probiotics were used in the GER-positive group than in the GER-negative group $(p<0.05)$. Although there was no difference in BPD between the two groups, severe BPD occurred more often in the GER-positive group than in the GER-negative group $(p<0.05)$.

Table 3. Risk factors of GER negative group and GER positive group 


\begin{tabular}{|c|c|c|c|c|}
\hline Clinical features & $\begin{array}{l}\text { GER negative } \\
(27)\end{array}$ & $\begin{array}{l}\text { GER positive } \\
(34)\end{array}$ & $t / x^{2}$ & $P$ \\
\hline female, n (\%) & $12(44.4)$ & $16(47.1)$ & 0.041 & 0.839 \\
\hline GA (weeks) & $29.96 \pm 1.41$ & $29.96 \pm 1.26$ & -0.001 & 0.999 \\
\hline BW $(g)$ & $1371 \pm 327$ & $1439 \pm 390$ & -0.733 & 0.467 \\
\hline Apgar score at $1 \mathrm{~min}$ & $7.59 \pm 2.08$ & $7.88 \pm 1.75$ & -0.59 & 0.557 \\
\hline PMA at full oral feeds (weeks) & $36.26 \pm 1.45$ & $35.18 \pm 2.16$ & 2.851 & 0.006 \\
\hline PMA at study (weeks) & $35.71 \pm 1.86$ & $35.21 \pm 1.59$ & 0.939 & 0.352 \\
\hline durations of PPN (days) & $27.37 \pm 13.67$ & $22.68 \pm 15.50$ & 1.237 & 0.221 \\
\hline caffeine administration (days) & $17.89 \pm 10.5$ & $12.35 \pm 11.04$ & 1.988 & 0.051 \\
\hline duration of mechanical ventilation (days) & $7.41 \pm 10.5$ & $3.76 \pm 5.5$ & 1.742 & 0.087 \\
\hline $\begin{array}{l}\text { duration of noninvasive auxiliary } \\
\text { ventilation (days) }\end{array}$ & $14.67 \pm 9.1$ & $8.71 \pm 7.9$ & 2.740 & 0.008 \\
\hline length of hospital stay (days) & $52.44 \pm 16.81$ & $48.11 \pm 22.55$ & 0.83 & 0.410 \\
\hline probiotics, n (\%) & $17(63.0)$ & $12(35.3)$ & 4.620 & 0.032 \\
\hline BPD, n (\%) & $8(29.6)$ & $13(38.2)$ & 0.494 & 0.482 \\
\hline severe $\mathrm{BPD}, \mathrm{n}(\%)$ & $2(7.4)$ & $10(29.4)$ & 4.611 & 0.032 \\
\hline NEC, n (\%) & $3(11.1)$ & 0 & & $0.081 *$ \\
\hline nosocomial infection, n (\%) & $6(22.2)$ & $7(20.6)$ & 0.024 & 0.877 \\
\hline
\end{tabular}

*Calculated by Fisher's Exact Test

GA: gestational age, BW: birth weight, PMA: postmenstrual age, PPN: partial parenteral nutrition, BPD: bronchopulmonary dysplasia, NEC: necrotizing enterocolitis.

A binary logistic regression analysis using caffeine administration, duration of mechanical ventilation, duration of noninvasive auxiliary ventilation, probiotics, severe BPD, and NEC (univariate $p<0.1$ ) revealed that delayed full oral feeds and probiotics could decrease the GER risk (OR, 0.642; $95 \% \mathrm{Cl}, 0.457-0.901$, $\mathrm{p}=0.01 ; \mathrm{OR}, 0.234,95 \% \mathrm{Cl}, 0.069-0.794, \mathrm{p}=0.02)$. Severe BPD increased the GER risk $(\mathrm{OR}, 6.890 ; 95 \% \mathrm{Cl}$, 1.125-42.209; $p=0.037$ ) (Table 4).

\section{Discussion}

As a physiologic phenomenon, GER is especially common in preterm infants. A 3-year retrospective study of 18,567 premature infants at 33 medical centers in North America found that the morbidity of GERD was as high as $10.3 \%$ for premature infants and that the lower the weight, the higher the morbidity (11). 
The diagnosis of GERD is difficult when based only on nonspecific clinical symptoms. In this study, we found that the prevalence of GER for preterm infants was $56 \%$, and there was significant clinical suspicion of reflux. Jadcherla et al. found that the prevalence of GER was $54 \%$; symptoms associated with GER were observed in 30 symptomatic infants with acid reflux $(48.5 \%)$ and nonacid reflux $(51.5 \%)$ (12). These results were similar to those of our study. Funderburk et al. reported that the prevalence of GER detected by $\mathrm{pH}-\mathrm{MII}$ monitoring was only $10 \%$ for symptomatic term and preterm infants (13), and that irritability, bradycardia, and desaturation were common signs and symptoms, which was not in accordance with our results. These differences may have been caused by the different subjects and clinical symptoms. In this study, we also found that most cases existed in infants with a GA of 30-32 weeks and BW more than $1500 \mathrm{~g}$, which is in accordance with the prevalence in North America (11). The reason was not clear and was thought to be due to a prematurity-associated decrease in acid production.

Infants born before 32 weeks of gestation are prone to apnea because of an immature respiratory center, whereas apnea triggered by feeding may be likely GER-related. Hypoxemia post-feeding may also be due to incoordination between feeding and breathing, immature laryngeal chemoreflex, or diaphragmatic fatigue. In Australia and the United States, almost half of all board-certified neonatologists believe that apnea is caused by GERD and are likely to recommend acid suppressors $(14,15)$. Cresi et al. found that the associations of GER and cardiorespiratory events, such as apnea existed in $12 \%$ of infants and most of them were caused by nonacidic reflux events (6). However, some studies speculated that there is no relationship between apnea and GER in preterm infants $(16,17)$. Nobile et al. studied 47 infants and found that only $11 \%$ of infants with GER had apnea or cyanosis; moreover, weakly acid refluxes occurred more often (18). Although it is commonly thought that GER could cause apnea, some studies of humans have concluded that apnea precedes a reflux episode and that reflux does not cause apnea $(19,20)$. In this study, we could not analyze the causal association between asphyxia and apnea. In our study, as a common phenomenon for both GER-negative and GER-positive infants, apnea affected nearly half of them (52.9\%). It has been reported that the prone position helps alleviate obstructive apnea (21). This mechanism may have decreased the occurrence of GER. Some studies indicated that weakly acidic reflux events, but not acid reflux events, are more prevalent in premature infants (22). Shin et al. studied 23 infants suspected of having GERD based on $\mathrm{pH}-\mathrm{MII}$ monitoring and found that the main symptom was apnea, with acid flux accounting for only $40.8 \%$ of all episodes (23); our results are similar to theirs.

Caffeine was often used to prevent BPD or apnea in very premature infants (24). Interestingly, we also found that infants with GER had less caffeine usage, although there was no difference between the GERpositive and GER-negative groups. The reason may be that caffeine delayed the gastric emptying time of preterm infants, which was verified according to ultrasound assessments (25).

Low BW preterm infants unable to feed orally must be fed via an intragastric tube for varying lengths of time. There was no difference in the effect on RI between small-bore and large-bore nasogastric (NG) tubes (26). It was also reported that there was no significant difference in acid exposure, whether with or without the NG tube. However, preterm infants without an NG tube had significantly more reflux events regardless of GA and BW (27). In the study, no association was found between earlier oral feeding and 
acid reflux. However, it was found that delayed oral feeding could decrease the risk of GER. The reason may be that the infants could tolerate and accommodate feedings better with the progress of postnatal corrected age. Therefore, we recommend not removing the gastric tube prematurely.

Traditionally, probiotics were thought to prevent gastrointestinal disorders, such as acute gastroenteritis, antibiotic-associated diarrhea, or necrotizing enterocolitis (28). However, no broad consensus exists to recommend the use of probiotics in the prevention of GER. A systematic review mentioned that 11 of 14 studies (79\%) reported positive efficacy of probiotics on reflux symptoms in adults (29). A prospective, multicenter, double-masked, placebo-controlled randomized clinical trial found that Lactobacillus reuteri (L. reuteri) DSM 17938 for 3 months reduced the onset of functional gastrointestinal disorders including GER in term neonates (30). Using real-time ultrasound imaging, L. reuteri DSM 17938 was confirmed efficient in improving reflux by accelerating gastric emptying (31). Thus, in our study, the probiotics used was $L$. reuteri, and we found that it effectively prevents the occurrence of GER. However, the role of $L$. reuteri still needs to be studied in a large-sample, multicenter clinical study.

The potential interrelationship of GER events is relevant not only to probiotics, but also to severe BPD. In our study, there was no relationship between GER and BPD or necrotizing enterocolitis or nosocomial infection. However, severe BPD poses a potential risk factor for GER. To date, the relationship between GER and BPD is controversial. GERD reportedly causes a significant increase in both BPD and NEC, thus leading to increased costs because of the resultant prolonged hospital stay (11). In a prospective observational cohort study that enrolled a total of 46 preterm infants born before 32 weeks of gestation and suspected of having GERD, infants with BPD had increased pH-only events and a corresponding SSI, probably because of the lower milk intake, impaired esophageal motility, or a peculiar autonomic nervous system response pattern. There was no difference in morbidity due to GER or clinical characteristics of the two groups, irrespective of the presence of BPD (32).

The limitations of the study include the limited number of cases and the retrospective observational study design; therefore, further prospective studies are needed to confirm our results. Finally, GERD in preterm infants is often over diagnosed by the present method of 24-hour pH monitoring. Despite these shortcomings, the benefits of the treatment of GER in symptomatic preterm infants warrant further technical refinement and wide application.

\section{Methods}

\section{Materials and Methods}

This retrospective cohort study involved infants born at 32 weeks of gestation or earlier at a regional tertiary care neonatal intensive care unit between November 2016 and November 2019. The inclusion criteria were gestational age (GA) $\leq 32$ weeks and clinical symptoms indicative of GER (including apnea, cyanosis, recurrent vomiting, aspiration pneumonia, and poor weight gain) according to the criteria of the North American Society for Pediatric Gastroenterology and Nutrition (5). Apnea was defined as breathing pauses lasting for $>20 \mathrm{~s}$, or for $>10 \mathrm{~s}$ together with bradycardia or oxygen desaturation (6). Cyanosis was 
judged by blood oxygen desaturation below $80 \%$. The exclusion criteria included digestive system abnormalities, congenital heart disease, neurological disorders, inherited metabolic disease, cow milk allergy, and death (7).

A total of 61 infants with clinical symptoms consistent with GERD whose parents agreed to gastric and esophageal 24-hour pH-MII monitoring were enrolled in this study. No surgeries were performed for GER. Written informed consent was obtained from the parents of all included patients. The study was approved by the Ethics Committee of the Shengjing Hospital of China Medical University (protocol no. 2017PS084J).

\section{Equipment for gastric and esophageal 24-hour pH-MII monitoring}

A pH monitor (Digitrapper ${ }^{\text {TM }} \mathrm{PH}-\mathrm{Z}$; Medtronic Inc., Minneapolis, MN, USA) was connected with a probe containing one $\mathrm{pH}$ channel and six impedance channels (Z1-Z6; Kanglian, Beijing, China). The probe was a single silicone probe, and the catheter consisted of monocrystalline antimony electrodes. The probes were calibrated before each study. Data were evaluated using the manufacturer's software (Reflux Reader v6.1 Software Updates) after each study.

All infants underwent ambulatory 24-h pH-MII measurements performed with gastric and esophageal probes connected to the device when they could ingest milk via breastfeeding or bottle. During the study, all infants breathed spontaneously, received an intermittent bottle, were not administered orogastric feeds, antireflux or antacid medications, inhaled medications, or diuretics. Infants were placed supine during monitoring. The neonatal $\mathrm{pH}$ probe was orally inserted into the esophagus. The esophageal probe position was verified by chest radiography performed 2 to $3 \mathrm{~cm}$ proximal to the LES. According to the Strobel formula, the probe length $(\mathrm{cm})$ was $0.87 \times(0.252 \times$ height +5$)(4)$. The gastric probe was placed on the fundus of the stomach. Both the esophageal $\mathrm{pH}$ and gastric $\mathrm{pH}$ were monitored. Each tracing was manually reviewed and validated by the same operator. Patients were monitored for 24 hours. Nurses documented the feeding times and symptoms during the observation period.

\section{Definition of reflux episodes}

GER was diagnosed based on the proximal pH channel. Reflux index (RI), which is the percentage of the total recording time with an esophageal $\mathrm{pH}<4$, was used to categorize pathological GER. Acid reflux was defined as abnormal when the RI was $\geq 7 \%$, irrespective of the impedance value (8). The esophageal liquid caused a decrease in impedance that persisted as long as the bolus was present between the two electrodes and returned to baseline when the liquid was cleared (9). In the present study, weakly acidic reflux was defined as pH between 4 and 7 and impedance values that decreased to $>20 \%$ of the baseline value. The distal $\mathrm{pH}$ channel showed that the $\mathrm{pH}$ was $<4$ in the stomach for more than $50 \%$ of $24 \mathrm{~h}$. Nonacidic GER was diagnosed when the RI was $<7 \%$, the impedance values decreased, and the intragastric $\mathrm{pH}$ was $<4$ during less than $50 \%$ of the total $24 \mathrm{~h}$ (10). For each $\mathrm{pH}-\mathrm{Mll}$ examination, the number of reflux episodes, duration of the longest reflux event, reflux events lasting $>5$ minutes, symptom index, symptom sensitivity index (SSI), symptom-associated probability (SAP) were also evaluated. 


\section{Statistical analysis}

Continuous data were analyzed using the independent samples $t$ test, and the results are presented as means \pm standard deviations. Noncontinuous data were assessed using the chi-square test and Fisher's exact test. Univariate variables with $p<0.1$ were introduced in a binary logistic regression analysis using the Forward Wald method. Adjusted odds ratios (OR) and corresponding 95\% confidence intervals (Cl) were estimated for all variables. All statistical analyses were performed using SPSS version 20.0 (SPSS Inc., Chicago, IL, USA). $\mathrm{P}<0.05$ was considered significanT.

\section{Declarations}

Conflicts of Interest: The authors declare that the research was conducted without any commercial or financial relationships that could be construed as a potential conflict of interest.

Author Contributions: Yu Hu collected and analyzed the clinical data. JianHua Fu designed the study. ShuCheng Zhang drafted and revised the manuscript. All authors read and approved the final manuscript.

Funding: National Natural Science Foundation of China $(30700917,81570465)$ provided funding for the development of the research and manuscript.

\section{References}

1. Czinn, S. J. \& Blanchard, S. Gastroesophageal reflux disease in neonates and infants: when and how to treat. Paediatr Drugs. 15, 19-27 (2013).

2. Rosen, R., et al. Pediatric gastroesophageal reflux clinical practice guidelines: joint cecommendations of the north American society for pediatric gastroenterology, hepatology, and nutrition and the European society for pediatric gastroenterology, hepatology, and nutrition. J Pediatr Gastroenterol Nutr. 66, 516-554 (2018).

3. Mitchell, D. J., McClure, B. G. \& Tubman, T. R. Simuhaneous monitoring of gastric and oesophageal $\mathrm{pH}$ reveals limitations of conventional oesophageal $\mathrm{pH}$ monitoring in milk fed infants. Arch Dis Child. 84, 273-276 (2001).

4. Shin, M. S. Esophageal $\mathrm{pH}$ and combined impedance-pH monitoring in children. Pediatr Gastroenterol Hepatol Nutr. 17, 13-22 (2014).

5. Rudolph, C. D., et al. Guidelines for evaluation and treatment of gastroesophageal reflux in infants and children: recommendations of the north American society for pediatric gastroenterology and nutrition. J Pediatr Gastroenterol Nutr. 32 Suppl 2, S1-S31 (2001).

6. Cresi, F., et al. Cardiorespiratory events in infants with gastroesophageal reflux symptoms: Is there any association? Neurogastroenterol Motil. 30, e13278 (2018).

7. Papachrisanthou, M. M. \& Davis, R. L. Clinical practice guidelines for the management of gastroesophageal reflux and gastroesophageal reflux disease: birth to 1 year of age. $J$ Pediatr Health 
Care. 29, 558-564 (2015).

8. Collins, C. R., Hasenstab, K. A., Nawaz, S. \& Jadcherla, S. R. Mechanisms of aerodigestive symptoms in infants with varying acid reflux index determined by esophageal manometry. J Pediatr. 206, 240247 (2019).

9. Fike, F. B., Mortellaro, V. E., Pettiford, J. N., Ostlie, D. J. \& St Peter, S. D. Diagnosis of gastroesophageal reflux disease in infants. Pediatr Surg Int. 27, 791-797 (2011).

10. Zerbib, F., et al. Normal values and day-to-day variability of 24-h ambulatory oesophageal impedance-pH monitoring in a Belgian-French cohort of healthy subjects. Aliment Pharmacol Ther. 22, 1011-1021 (2005).

11. Jadcherla, S. R., et al. Practice variance, prevalence, and economic burden of premature infants diagnosed with GERD. Hosp Pediatr. 3, 335-341 (2013).

12. Suárez-Morán, E., et al. Influence of central apnea in the preterm newborn with gastroesophageal reflux disease. Cir Cir. 79, 511-519 (2011).

13. Funderburk, A., et al. Temporal association between reflux-like behaviors and gastroesophageal reflux in preterm and term infants. J Pediatr Gastroenterol Nutr. 62, 556-561 (2016).

14. Golski, C. A., et al. Pediatric specialists' beliefs about gastroesophageal reflux disease in premature infants. 125, 96-104 (2010).

15. Abu Jawdeh, E. G., \& Martin, R. J. Neonatal apnea and gastroesophageal reflux (GER): is there a problem? Early Hum Dev. 89 Suppl 1, S14-S16 (2013).

16. Poets, C. F. Apnea of prematurity: what can observational studies tell us about pathophysiology. Sleep Med. 11, 701-707 (2010).

17. Molloy, E. J., Di Fiore, J. M. \& Martin, R. J. Does gastroesophageal reflux cause apnea in preterm infants? Biol Neonate. 87, 254-261 (2005).

18. Nobile, S., Marchionni, P., Noviello, C. \& Carnielli, V. P. Correlation between cardiorespiratory events and gastro-esophageal reflux in preterm and term infants: analysis of predisposing factors. Early Hum Dev. 134, 14-18 (2019).

19. Di Fiore, J., Arko, M., Herynk, B., Martin, R., \& Hibbs, A. M. Characterization of cardiorespiratory events following gastroesophageal reflux in preterm infants. J Perinatol. 30, 683-687 (2010).

20. Slocum, C., Arko, M., Di Fiore, J., Martin, R. J., \& Hibbs, A.M. Apnea, bradycardia and desaturation in preterm infants before and after feeding. J Perinatol. 29, 209-212 (2009).

21. Bhat, R. Y., et al. Effect of prone and supine position on sleep, apneas, and arousal in preterm infants. 118, 101-107 (2006).

22. Magistà, A. M., et al. Multichannel intraluminal impedance to detect relationship between gastroesophageal reflux and apnoea of prematurity. Dig Liver Dis. 39, 216-221 (2007).

23. Shin, M. S., et al. Impedance-pH monitoring and conventional pH monitoring are complementary methods to detect association between gastroesophageal reflux and apnea-related symptoms in preterm infants and neonates. J Matern Fetal Neonatal Med. 25, 2406-2410 (2012). 
24. Lodha, A., et al. Association of early caffeine administration and neonatal outcomes in very preterm neonates. JAMA Pediatr. 169, 33-38 (2015).

25. Gounaris, A. K., et al. Caffeine and gastric emptying time in very preterm neonates. J Clin Med. 9, E1676 (2020).

26. Dotson, R. G., Robinson, R. G. \& Pingleton, S. K. Gastroesophageal reflux with nasogastric tubes. Effect of nasogastric tube size. Am J Respir Crit Care Med. 149, 1659-1662 (1994).

27. Murthy, S. V., et al. Nasogastric feeding tubes may not contribute to gastroesophageal reflux in preterm infants. Am J Perinatol 35,643-647 (2018).

28. Cheng, J. \& Ouwehand, A. C. Gastroesophageal reflux disease and probiotics: a systematic Review. Nutrients. 12, 132 (2020).

29. Perceval, C., Szajewska, H., Indrio, F., Weizman, Z. \& Vandenplas, Y. Prophylactic use of probiotics for gastrointestinal disorders in children. Lancet Child Adolesc Health. 3, 655-662 (2019).

30. Indrio, F., et al. Prophylactic use of a probiotic in the prevention of colic, regurgitation, and functional constipation: a randomized clinical trial. JAMA Pediatr. 168, 228-333 (2014).

31. Indrio F, Riezzo G, Raimondi F, et al. Lactobacillus reuteri accelerates gastric emptying and improves regurgitation in infants. Eur J Clin Invest. 41, 417-422 (2011).

32. Nobile, S., et al. Are infants with bronchopulmonary dysplasia prone to gastroesophageal reflux? a prospective observational study with esophageal pH-impedance monitoring. J Pediatr. 167, 279-285 (2015). 